\title{
Meson spectroscopy with derivative quark sources
}

\author{
Christof Gattringer ${ }^{a}$, Leonid Ya. Glozman ${ }^{a}$, C. B. Lang ${ }^{a}$, Daniel Mohler ${ }^{* a}$ \\ and Sasa Prelovsek ${ }^{b}$ \\ ${ }^{a}$ Institut für Physik, FB Theoretische Physik, Universität Graz, A-8010 Graz, Austria \\ ${ }^{b}$ University of Ljubljana and Institute Josef Stefan, 1000 Ljubljana, Slovenia \\ E-mail: christof.gattringereuni-graz.at leonid.glozmaneuni-graz.at, \\ christian.langeuni-graz.at daniel.mohler@uni-graz.at, \\ Sasa.Prelovsekeijs.si
}

\begin{abstract}
We present results for masses of light mesons obtained with the variational method using an enhanced basis of interpolating field operators with different quark smearings. The interpolators are constructed from Jacobi-smeared quarks of a Gaussian type as well as from derivative quark sources obtained by a covariant derivative acting on the Gaussian sources. For our analysis we use quenched gauge configurations with Chirally Improved quarks and the Lüscher-Weisz gauge action on a $16^{3} \times 32$ lattice with $a=0.148 \mathrm{fm}$. We discuss the influence of derivative sources on the overlap with excited states.
\end{abstract}

The XXV International Symposium on Lattice Field Theory

July 30 - August 42007

Regensburg, Germany

\footnotetext{
* Speaker.
} 


\section{Introduction}

Lattice QCD opened the possibility of providing a model independent ab initio calculation of the QCD mass spectrum. Extracting signals from excited states has however proven to be a formidable task. A previous study of the BGR-Collaboration [1] employed the variational technique [2, 3] to study excited mesons on the lattice using standard meson interpolators with Gaussiansmeared sources and sinks.

For an accurate representation of excited states in the variational approach it is crucial to use a basis with good overlap with the physical states. We therefore construct additional meson-interpolators with derivative sources obtained by a covariant derivative acting on the Jacobi smeared sources and explore their effect in the variational approach.

\section{Details of the calculation}

\subsection{The variational method}

We use a basis of interpolators $O_{i}, i=1, \ldots, N$ with the quantum numbers of the desired states (projected to zero momentum) and compute the matrix of cross correlations:

$$
C(t)_{i j}=\left\langle O_{i}(t) \overline{O_{j}}(0)\right\rangle=\sum_{n}\left\langle 0\left|O_{i}\right| n\right\rangle\left\langle n\left|O_{j}^{\dagger}\right| 0\right\rangle e^{-t M_{n}} .
$$

It can be shown [3] that the solutions to the generalized eigenvalue problem

$$
C(t) \vec{v}_{i}=\lambda_{i}(t) C\left(t_{0}\right) \vec{v}_{i}
$$

behave as

$$
\lambda_{i}(t) \propto e^{-t M_{i}}\left(1+\mathscr{O}\left(e^{-t \Delta M_{i}}\right)\right)
$$

where $\Delta M_{i}$ is the mass difference between the state $i$ and the closest lying state.

Therefore, up to the given order, the largest eigenvalue decays with the mass of the ground state, the second largest eigenvalue with the mass of the first excited state and so on. For an accurate description, the interpolators should be linearly independent, as orthogonal as possible and possess a strong overlap with physical states. The task will be to construct new interpolators which are independent of the standard interpolators and have a good overlap with excited states.

\subsection{Effective masses and eigenvectors}

To display the results, one commonly plots the effective masses determined from ratios of eigenvalues

$$
a M_{i, \mathrm{eff}}\left(t+\frac{1}{2}\right)=\ln \left(\frac{\lambda_{i}(t)}{\lambda_{i}(t+1)}\right) .
$$

A fit to the exponential decay of the eigenvalues is then performed in the time interval where the effective mass plot shows a plateau. As a consistency check, the entries of the corresponding eigenvectors should also show plateaus and can serve as a fingerprint for the "wave functions". To deal 
with eigenvectors that are orthonormal we calculate the eigenvectors of the modified eigenvector problem

$$
C\left(t_{0}\right)^{-\frac{1}{2}} C(t) C\left(t_{0}\right)^{-\frac{1}{2}} \vec{v}_{i}^{\prime}=\lambda_{i}(t) \vec{v}_{i}^{\prime}
$$

(This assumes $C\left(t_{0}\right)$ to be positive definite.)

\subsection{Smeared sources and sinks}

The first step for all our sources is Jacobi smearing [4, 5] of point sources $s_{0}$ located at timeslice $t=0$ :

$$
\begin{aligned}
s_{0}^{(\alpha, a)}(\vec{y}, 0)_{\rho, c} & =\delta(\vec{y}, 0) \delta_{\rho \alpha} \delta_{c a}, \\
s^{(\alpha, a)} & =\sum_{n=0}^{N} \kappa^{n} H^{n}, \\
H(\vec{x}, \vec{y}) & =\sum_{i=1}^{3}\left(U_{i}(\vec{x}, 0) \delta(\vec{x}+\hat{i}, \vec{y})+U_{i}(\vec{x}-\hat{i}, 0)^{\dagger} \delta(\vec{x}-\hat{i}, \vec{y})\right) .
\end{aligned}
$$

In [1] two different parameter values for $\kappa$ and $N$, giving rise to wide and narrow sources $\left(S_{w}, S_{n}\right)$, have been used. In addition, we now construct covariant derivatives which act upon a wide smeared source to form our derivative quark sources $W_{d_{i}}$ :

$$
\begin{aligned}
P_{i}(\vec{x}, \vec{y}) & =U_{i}(\vec{x}, 0) \delta(\vec{x}+\hat{i}, \vec{y})-U_{i}(\vec{x}-\hat{i}, 0)^{\dagger} \delta(\vec{x}-\hat{i}, \vec{y}), \\
W_{d_{i}} & =P_{i} S_{w} .
\end{aligned}
$$

With these sources, meson interpolators of definite quantum numbers are constructed.

\subsection{Interpolators used}

Table 1 shows the interpolators used for different meson channels. The ones labeled "old interpolators" are those of [1], while the "new interpolators" contain at least one derivative. The total number of interpolators as given in the last column can be obtained by combining all nondegenerate combinations of the different smearings with all possible Dirac structures.

In some cases, an (anti-) symmetrization of the interpolators is necessary to obtain the correct behavior under charge conjugation. Therefore, interpolators denoted as $\bar{u}_{d_{i}} \Gamma d_{n / w}$ in the table should be read as $\bar{u}_{d_{i}} \Gamma d_{n / w}-\bar{u}_{n / w} \Gamma d_{d_{i}}$. We restrict ourselves to light, isovector $(I=1)$ mesons with degenerate quark masses $m_{u}=m_{d}$.

\subsection{Technicalities}

For our analysis we used 99 uncorrelated quenched gauge configurations with Chirally Improved (CI) [8, 7] quarks and the Lüscher-Weisz gauge action [9]. We work on a $16^{3} \times 32$ lattice with $a=0.148 \mathrm{fm}$ determined from [10] the Sommer parameter. We study several quark mass parameters in the range $a m_{q}=0.02 \ldots 0.2$. All errors we quote are statistical errors determined with the jackknife method. 


\begin{tabular}{|c|c|c|c|c|c|c|c|}
\hline & $J^{P C}$ & \multicolumn{2}{|c|}{ old interpolators } & \multicolumn{2}{|c|}{ additional, new interpolators } & $\sharp$ \\
\hline pseudoscalar & $0^{-+}$ & $\bar{u}_{n / w} \gamma_{5} d_{n / w}$ & $\bar{u}_{n / w} \gamma_{4} \gamma_{5} d_{n / w}$ & $\bar{u}_{d_{i}} \gamma_{i} \gamma_{4} \gamma_{5} d_{n / w}$ & $\bar{u}_{d_{i}} \gamma_{5} d_{d_{i}}$ & $\bar{u}_{d_{i}} \gamma_{4} \gamma_{5} d_{d_{i}}$ & 10 \\
\hline scalar & $0^{++}$ & $\bar{u}_{n / w} d_{n / w}$ & & $\bar{u}_{d_{i}} \gamma_{i} d_{n / w}$ & $\bar{u}_{d_{i}} \gamma_{i} \gamma_{4} d_{n / w}$ & $\bar{u}_{d_{i}} d_{d_{i}}$ & 8 \\
\hline vector & $1^{--}$ & $\bar{u}_{n / w} \gamma_{i} d_{n / w}$ & $\bar{u}_{n} \gamma_{i} \gamma_{4} d_{n / w}$ & $\bar{u}_{d_{i}} d_{n / w}$ & $\bar{u}_{d_{i}} \gamma_{k} d_{d_{i}}$ & & 9 \\
\hline pseudovector & $1^{++}$ & $\bar{u}_{n / w} \gamma_{i} \gamma_{5} d_{n / w}$ & & $\bar{u}_{d_{i}} \gamma_{k} \gamma_{5} d_{d_{i}}$ & & & 4 \\
\hline
\end{tabular}

Table 1: Meson interpolators; $n / w$ denote narrow and wide Jacobi smearing and $d_{i}$ stands for a derivative source in the $i$-direction. The last column shows the total number of different interpolators.

\section{Results}

To get an idea about the properties of the correlators, we first plot the normalized diagonal entries of the correlation matrices for the pseudoscalar and vector mesons. In figure 1 the simple interpolators have been colored blue, while the new correlators from derivative sources appear in red. As can be seen from the behavior at small euclidean times $t$, the new correlators have stronger contributions from excited states, i.e., they start out with a steeper slope. However, all correlators are dominated by the ground state at large time separations.

Figure 2 shows a comparison of selected effective mass plateaus from the optimal set of old interpolators compared to the new results (obtained from the optimal combination of both old and new interpolators) at different values of the quark mass. Plateaus are also found in the components of the corresponding eigenvectors.

For the pseudoscalar mesons (graphs on the upper 1.h.s.) we obtain improved results for the excited states at all quark masses. Both the old and the new sets are obtained with a basis of four interpolators. This shows that the observed effect does not just stem from simply enlarging the basis. Some interpolators mainly contribute noise without significantly enhancing the overlap to the physical states. Looking at the modified eigenvectors of equation (2.5) as depicted in figure 3 , clear plateaus are observed and the new interpolators (second and third entries) mainly contribute to the second excited state which could not be observed using the old set of interpolators alone.

Similarily for the vector mesons (upper r.h.s. of figure 2), improved results are obtained for the excited states at all quark masses. Here, however, the new basis consists of six interpolators while the old basis only contains four. Both the ground state and newly observed third excited state are dominated by the old interpolators while the first and second excited states are dominated by the new interpolators. We also would like to note that the finer $(a \approx 0.12 \mathrm{fm})$ quenched lattice considered in [1] allowed for a cleaner separation of the first and second excited states.

For the scalar (lower 1.h.s. of figure 2) and pseudovector (lower r.h.s. of figure 2) mesons, there is a noticeable improvement of the ground state plateaus. The old graph for the pseudovectors stems from a basis of three interpolators while the new graph is obtained from just one correlator alone. The location of the plateau agrees within error bounds but the errors obtained from the new interpolator are considerably smaller. For the scalars, the plots have been obtained from a basis of three interpolators. Using derivative sources, plateaus can be extracted at small values of the quark mass where no plateau is observed in the old interpolators. For the quenched data, scalar 

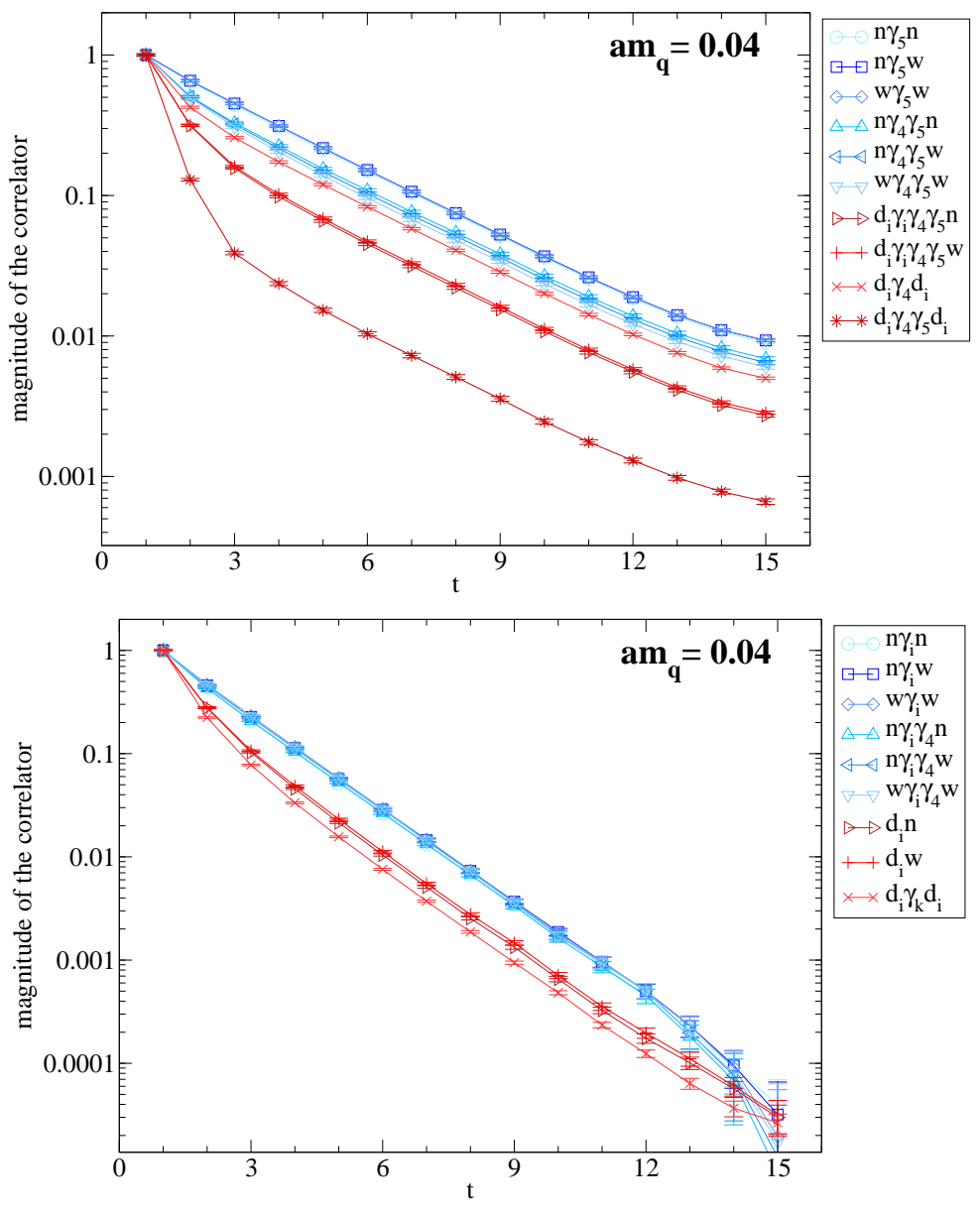

Figure 1: Diagonal correlators for the pseudoscalar (top) and vector (bottom) mesons at $a m_{q}=0.04 . n / w$ denote narrow and wide Jacobi smearing and $d_{i}$ stands for a derivative source in the $i$-direction.

correlators contain contaminations from ghost states and it has been shown, that the variational method can isolate such unphysical contributions [6]. Overall, ghost contributions to derivative interpolators seem to be smaller and some interpolators show almost no sign of such contributions. Where present, the variational method separates the ghosts from the physical signal and the ground state plateau can be clearly extracted (shown in figure 2). Concerning a full dynamical simulation, data from only a few dynamical configurations suggests that interpolators of the type $\bar{u}_{d_{i}} \gamma_{i} d_{n / w}$, which are important in the quenched case, seem to have very limited overlap in the dynamical case.

\section{Conclusions and upcoming investigations}

In this article we have presented first results for pseudoscalar, scalar, vector and pseudovector mesons with derivative quark sources and CI fermions on a quenched lattice. We employed the variational technique with both Jacobi-smeared sources of a Gaussian type as well as derivativetype sources.

It has been demonstrated that interpolators constructed with derivative quark sources lead to an enhanced signal for a variety of different meson channels. For pseudoscalar and vector mesons, the 


\section{pseudoscalar}
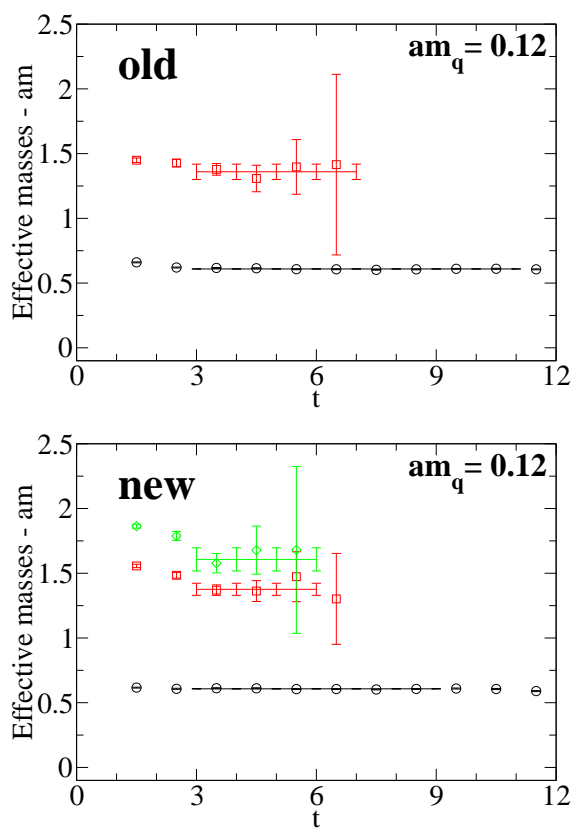

scalar
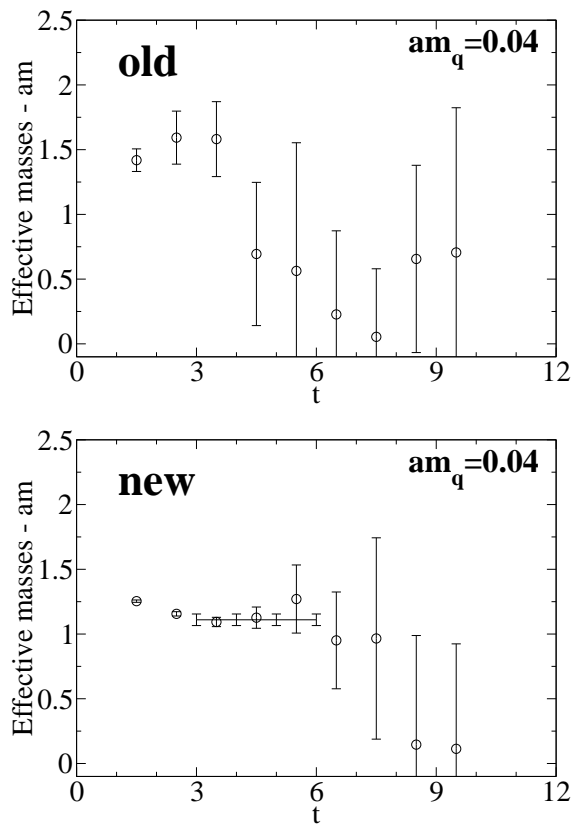

vector
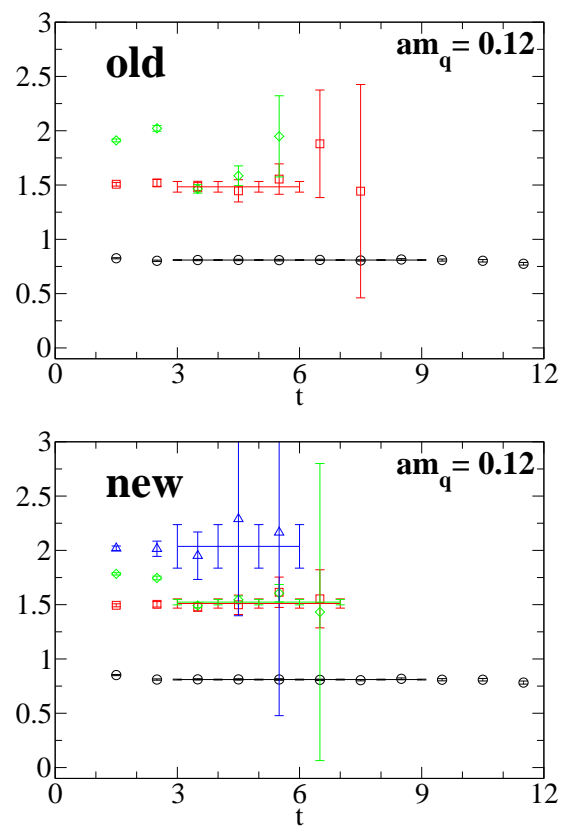

pseudovector
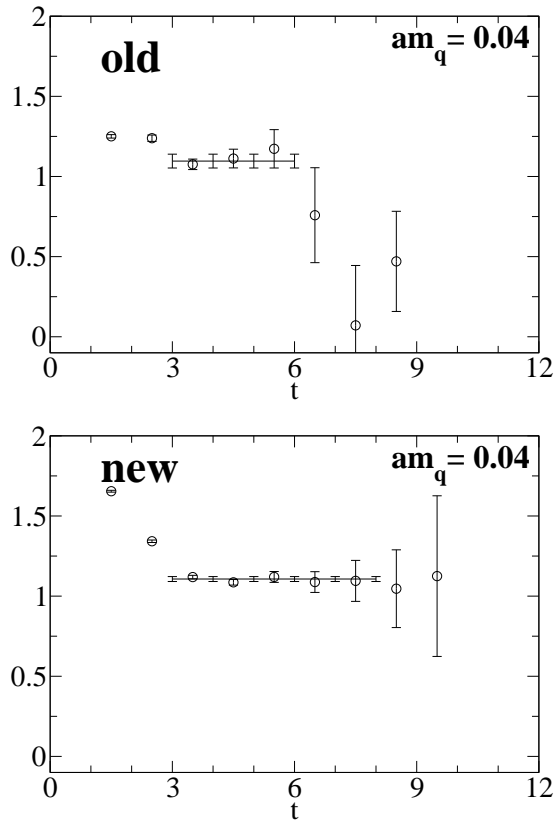

Figure 2: Effective mass plots for the pseudoscalar (upper left), vector (upper right), scalar (lower left) and pseudovector (lower right) mesons. We compare the results from the best selection of simple interpolators ("old") to the case where the new derivative interpolators are added ("new"). The horizontal lines indicate the fit ranges and their error bars indicate the statistical error of the fit. 

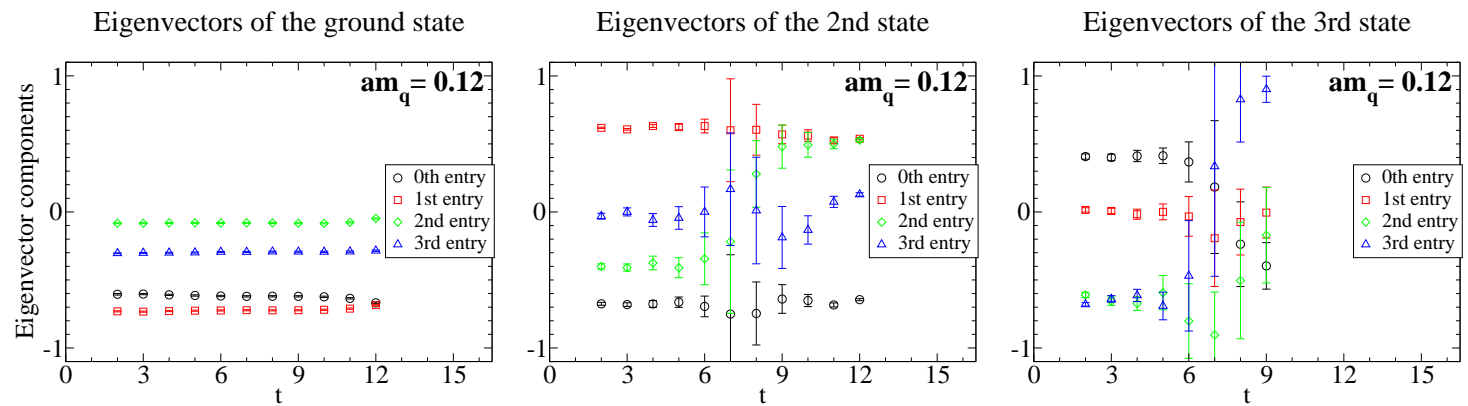

Figure 3: Eigenvector components of the modified eigenvector problem for the pion ground state (1.h.s. plot), first and second excited states (center and r.h.s. plot). The plots correspond to the effective mass plateaus shown in figure 2 .

correlators constructed from such interpolators display a significantly better overlap with excited states. In both cases excited states could be observed that were not found with the smaller basis. For the scalar and pseudovector mesons, overlap with the ground states can be improved which leads to ground state masses with significantly smaller statistical errors.

We have clearly demonstrated that derivative quark sources provide an essential enlargement of the basis of interpolators in excited meson spectroscopy. Although we are convinced that derivative interpolators will be an important tool also in the dynamical case, it is probably too early to claim a one to one correspondence of the interpolator content.

We are currently extending our calculations to dynamical configurations obtained in the BGR dynamical CI project. Furthermore, we investigate the construction of baryon interpolators from derivative quark sources and the effects of link smearing on our interpolators.

\section{Acknowledgments}

This work has been supported in part by the "Fond zur Förderung der wissenschaftlichen Forschung in Österreich" (FWF DK W1203-N08 and P19168-N16).

\section{References}

[1] T. Burch et al., Phys. Rev. D73, 094505 (2006) [hep-lat/ 0601026 ].

[2] C. Michael, Nucl. Phys. B259, 58 (1985).

[3] M. Lüscher and U. Wolff, Nucl. Phys. B339, 222 (1990).

[4] S. Güsken et al., Phys. Lett. B227, 266 (1989).

[5] C. Best et al., Phys. Rev. D56, 2743 (1997) [hep-lat/9703014].

[6] T. Burch et al., Phys. Rev. D73, 017502 (2006) [hep-lat/0511054].

[7] C. Gattringer, Phys. Rev. D63 114501 (2001) [hep-lat/ 0003005 ]

[8] C. Gattringer, I. Hip and C.B. Lang, Nucl. Phys. B597, 451 (2001) [hep-lat/ 0007042 ]

[9] M. Lüscher and P. Weisz, Commun. Math. Phys. 97, 59 (1985)

[10] C. Gattringer, R. Hoffmann and S. Schaefer, Phys. Rev. D65, 094503 (2002) [hep-lat/ 0112024 ] 